\title{
Editorial
}

\section{La atención y los cuidados a los supervivientes de cáncer en España: un reto pendiente de las políticas sanitarias}

\author{
Cristina García Vivar, Nuria Domenech-Climent*, Melinda González Concepción, Gema González \\ Monterubio, Vanesa Navarro Castuera, Catalina Rubio Uria, Ainhoa Ulibarri Ochoa \\ *Autor de correspondencia: Nuria Domenech-Climent. nuria.domenech@ua.es
}

\section{Grupo de trabajo Supervivientes de Cáncer. Sociedad Española de Enfermería Oncológica (SEEO)}

Cristina García Vivar. Departamento de Ciencias de la Salud. Universidad.

Pública de Navarra. IdiSNA - Instituto de Investigación Sanitaria de Navarra, Pamplona. ORCID: 0000-0002-6022-559X.

Nuria Domenech-Climent. Enfermera Departamento de Salud de Alcoy (Alicante). Profesora asociada del Departamento de Enfermería de la Universidad de Alicante.

Melinda González Concepción. Profesora Titular. Escuela Universitaria de Enfermería y Terapia Ocupacional de Terrassa. Barcelona. Universidad Autónoma de Barcelona.

Gema González Monterubio. Supervisora de Enfermería de Área Oncológia Médica, Hematología y Radioterapia. Hospital Universitario 12 de Octubre. Madrid.

Vanesa Navarro Castuera. Enfermera de Onco-Hematología del Hospital El Bierzo de Ponferrada (León).

Catalina Rubio Uria. Enfermera Hospital de día de Oncología. Hospital Universitario de Canarias.

Ainhoa Ulibarri Ochoa. Escuela Universitaria de Enfermería de Vitoria-Gasteiz. OsakidetzaServicio Vasco de Salud.

Los gobiernos europeos y de España han mostrado un interés creciente en la atención al cáncer como un objetivo político ${ }^{1-3}$. Tales intereses tienen potencial como respuesta a la alta incidencia y prevalencia del cáncer en Europa y en España, y la consecuente repercusión social y económica en términos de morbi-mortalidad $y$ costes para los sistemas sanitarios ${ }^{3}$.

En España, se prevé que el número de personas diagnosticadas de cáncer en el año 2020 sea de 277.394 (de los cuales el $40 \%$ son en personas mayores de 65 años), incidencia muy similar a la de 2019, según las estimaciones de la Red Española de Registros de Cáncer (REDECAN) ${ }^{4}$. Respecto a la prevalencia en España, los últimos datos ofrecidos por el registro mundial GLOBOCAN (Global Cancer Observatory) apuntan a una prevalencia a los 5 años de 772.853 casos, siendo los más prevalentes en varones el cáncer de próstata $(25,8 \%)$, colorrectal $(15,3 \%)$ y vejiga $(11,7 \%)$, y en mujeres, el cáncer de mama $(36,2 \%)$, colorrectal $(11,8 \%)$ y útero $(7,2 \%)^{5}$.

Respecto a la supervivencia desde el diagnóstico, las últimas cifras publicadas en enero de 2020 por REDECAN para el periodo 2008-2013, fueron del $70,9 \%, 55,9 \%$ y $48,9 \%$ en hombres a 1,3 y 5 años respectivamente, y del $77,4 \%, 66,6 \%$ y $61,7 \%$ en mujeres a 1,3 y 5 años respectivamente ${ }^{6}$. Es preciso señalar que existe variabilidad de la supervivencia según el tipo de tumor, el estadio de la enfermedad en el momento del diagnóstico y el grado de efectividad del tratamiento terapéutico. Así, por ejemplo, la supervivencia es baja para algunos tumores, como el cáncer de páncreas ( $10 \%$ en mujeres y $7 \%$ en hombres) o el de pulmón (18\% en mujeres y $13 \%$ en hombres). Por el contrario, la supervivencia más alta es para los 
cánceres de próstata con un $90 \%$ y de mama con un $86 \%$ de supervivencia en mujeres.

Se estima que la supervivencia se ha duplicado en los últimos 40 años y es probable que continúe aumentando en los próximos años. Este incremento es un indicador clave de la efectividad de la atención al cáncer en el sistema de salud español, especialmente para algunos cánceres. Sin embargo, los supervivientes pueden experimentar secuelas y efectos secundarios tardíos derivados de los tratamientos y del propio cáncer que en muchos casos impactan en su calidad de vida ${ }^{7}$. Los estudios llevados a cabo con población superviviente muestran que, por el hecho de haber padecido cáncer, las personas pueden tener necesidades físicas, psicosociales y de información 8-12, que a menudo no están cubiertas por el sistema sanitario ${ }^{13}$. Es por ello que organismos nacionales e internacionales insisten en la necesidad de ofrecer una atención específica para esta etapa de la trayectoria del cáncer, conocida como larga supervivencia de cáncer o supervivencia permanente ${ }^{14,15}$. Así por ejemplo, la Sociedad Española de Oncología Médica (SEOM) o la Sociedad Española de Enfermería Oncológica (SEEO) abogan por la creación de alianzas entre los especialistas en oncología y el equipo de Atención Primaria para el seguimiento de personas que han sido dadas de alta en oncología 14. Las corrientes más actuales, especialmente en Europa, promueven modelos de atención a la supervivencia desde la Atención Primaria ${ }^{16,17 .}$

Pero ¿cuál es la situación respecto a las políticas sanitarias en España para el abordaje de la supervivencia de cáncer? Para dar respuesta a esta pregunta, el grupo de trabajo de la SEEO "Supervivientes de cáncer", formado por enfermeras de distintas comunidades autónomas y con distintos perfiles (especialistas en oncología, investigadoras, docentes y gestoras), llevó a cabo una revisión de los planes y programas de salud publicados por las comunidades autónomas de España para identificar las iniciativas descritas para la coordinación y seguimiento de los largos supervivientes de cáncer. Los resultados de esta revisión han evidenciado un vacío significativo en los planes de salud de las distintas comunidades sobre la atención a la supervivencia. En particular, nuestra revisión arroja luces sobre la falta de abordaje sistematizado de los supervivientes de cáncer, es decir aquellas personas que han finalizado los tratamientos oncológicos y se encuentran libres de enfermedad tras un tiempo (más de 3 años) desde el diagnóstico.

Cierto es que existe alguna excepción como es el caso de Navarra con su "Estrategia de Prevención y Atención al Cáncer en Navarra 2014-2020"18, País Vasco con su "Plan Oncológico de Euskadi 2018-2023" 19, la Comunidad Valenciana con su "Estrategia contra el cáncer de la Comunitat Valenciana 2019-2022" 20 , Cataluña con su "plan director de Catalunya 2015-2020" 21 y Castilla y León con su "Estrategia de Atención al paciente oncológico en Castilla y León" (ONcyl) 2015-2019 22. No obstante, si bien en estos planes de salud y estrategias sanitarias existe una mención específica a la supervivencia, consideramos que las acciones son vagas y poco detalladas. El plan de salud es el instrumento primordial de la planificación en salud de un territorio, por el cual se constituyen las prioridades de intervención sanitaria, se establecen objetivos y se concretan los programas para mejorar los resultados en salud en una comunidad.

No podemos obviar que en España se están iniciando experiencias en la coordinación especializada-primaria para la atención a supervivientes. Como ejemplos podemos enumerar la 
consulta oncológica extrahospitalaria del Departamento ${ }^{9}$ de la Comunidad Valenciana; experiencias piloto en seguimiento de pacientes con diferentes tipos de cáncer por atención primaria con protocolos de seguimiento en Cataluña y Navarra; el caso de Castilla y León que ha iniciado un proyecto piloto de plan de atención a los largos supervivientes de cáncer, enmarcado dentro del programa de coordinación primaria-especializada en el que destaca la creación de una Unidad de Largos Supervivientes en Oncología; el taller "Cuidando mi salud después del cáncer" dentro del programa de Paciente Activo del País Vasco, o iniciativas de ámbito privado como las llevadas a cabo por la Fundación Sandra Ibarra que impulsó la creación de una Unidad de Supervivientes en el Hospital de Fuenlabrada, pero son iniciativas puntuales que no responden a un plan estratégico nacional o regional de atención a estos pacientes.

Estos datos muestran una realidad que ya fue identificada y publicada por primera vez en 2006 por el Instituto de Medicina de los Estados Unidos (National Institute of Medicine, NIM) en su informe "De paciente con cáncer a superviviente de cáncer: perdido en la transición (From cancer patient to cancer survivor: lost in transition) ${ }^{23}$. En él se reconocía la necesidad de mejorar la atención a los supervivientes a través de la implementación de un Plan de Cuidados del Superviviente (conocido en el contexto internacional como "Survivorship Care Plan") para ser compartido entre los niveles de asistencia secundaria y primaria.

Desde entonces, muchos países, especialmente Canadá, Estados Unidos y algunos países del norte de Europa han tratado de poner en marcha en sus respectivos sistemas sanitarios planes específicos para largos supervivientes de cáncer ${ }^{24}$. En España, también se está impulsando esta iniciativa, especialmente a raíz de la publicación por la SEOM del Plan Integral de Atención a los Largos Supervivientes de Cáncer" en $2013^{15}$. Sin embargo, la realidad es que existen iniciativas aisladas en algunas comunidades autónomas y por lo tanto la población de largos supervivientes no está recibiendo una atención que cubra sus necesidades de salud, al contrario de lo que sucede con otros programas de seguimiento al paciente crónico (diabetes, cardiovascular, etc).

Como enfermeras y con la visión de los cuidados integrales tanto a la persona superviviente como a la familia, no podemos dejar de señalar la necesidad de establecer un modelo de atención a la supervivencia de cáncer que vaya más allá de la efectividad de los tratamientos y donde se recoja la coordinación de los cuidados y necesidades de los supervivientes, tanto en el ámbito de la salud física y emocional como en el ámbito social, y dotar dicha atención de recursos humanos adecuados para llevarlos adelante. Enfermería juega aquí un papel importante y puede liderar, por su contacto directo con el paciente y la familia a lo largo de todo el proceso oncológico, la coordinación entre los diferentes recursos y niveles asistenciales, siendo capaz de dar respuesta a las diferentes necesidades (de información, de cuidados, complicaciones y secuelas, laborales, psicológicas...) que surjan durante el proceso y que, dentro de un equipo multidisciplinar, atienda y movilice los recursos que sean necesarios ${ }^{25}$.

Por todo ello, el objetivo de esta editorial es invitar a los gobiernos y consejerías de salud a invertir en supervivencia de cáncer. Instamos también a que investigadores, clínicos y gestores, de distintas disciplinas de la salud y niveles asistenciales, se unan para impulsar la implementación de planes de cuidados al superviviente de cáncer, instrumento que promueva la coordinación y seguimiento entre 
atención especializada y atención primaria. Para ello, se requiere una estrategia integral, multidisciplinar, coordinada entre niveles asistenciales y con un enfoque centrado en el bienestar y la calidad de vida del superviviente, y no sólo en datos epidemiológicos de morbi-mortalidad, que sin duda son necesarios para la toma de decisiones en salud, pero que en muchas ocasiones no reflejan las verdaderas necesidades de atención y cuidados de estas personas.

\section{Referencias:}

1. Ministerio de Sanidad y Política Social. Estrategia en Cáncer del Sistema Nacional de Salud.

https://www.mscbs.gob.es/organizacion/sns/pl anCalidadSNS/pdf/Actualizaci

EstrategiaCancer.pdf; 2010[consultada 15/09/2020].

2. Sant $M$, Chirlaque MD, Agresti $R$, et al. Survival of women with cancers of breast and genital organs in Europe 1999-2007: Results of the EUROCARE-5 study. Eur J Cancer 2015;51(15):2191-2205.

doi:10.1016/j.ejca.2015.07.022

3. Rossi S, Baili P, Capocaccia R, et al. The EUROCARE-5 study on cancer survival in Europe 1999-2007: Database, quality checks and statistical analysis methods. Eur J Cancer 2015;51(15):2104-19.

doi:10.1016/j.ejca.2015.08.001

4. Red Española de registros de Cáncer (REDECAN). REDECAN, estadísticas 2020, https://redecan.org/redecan.org/es/index.html ; 2020[consultada 15/09/2020].

5. Sociedad Española de Oncología Médica (SEOM). Las Cifras Del Cáncer En España 2020, https://seom.org/seomcms/images/stories/rec ursos/Cifras del cancer 2020.pdf. :

2020 [consultada 15/09/2020].

6. Red Española de registros de Cáncer (REDECAN). Supervivencia de Cáncer En España, 2002-2013,

https://redecan.org/redecan.org/es/Informe_S upervivencia_REDECAN_2020.pdf; 2020 [consultada 15/09/2020].

7. Cavers D, Habets L, Cunningham-Burley S, et al. Living with and beyond cancer with comorbid illness: a qualitative systematic review and evidence synthesis. J Cancer Surviv 2019;13(1):148-59. doi:10.1007/s11764-0190734-z

8. Vivar C. Impacto psicosocial del cáncer de mama en la etapa de larga supervivencia: propuesta de un plan de cuidados integral para supervivientes. Aten Primaria 2012;44(5):28892. ISSN 0212-6567

9. Bilodeau K, Tremblay D, Durand MJ. Gaps and delays in survivorship care in the return-towork pathway for survivors of breast cancer-a qualitative study. Curr Oncol 2019;26(3):e414e417. doi: $10.3747 / \mathrm{co} .26 .4787$

10. Barragan $B$, Jovell $A J$. Informe sobre las necesidades de los supervivientes de cáncer Madrid: GEPAC-Grupo Español de Pacientes con Cáncer; $2012 . \quad$ Disponible en: http://www.gepac.es/docs/informe_supervivie ntes.pdf

11. O'Connor M, O'Donovan B, Drummond F, et al. National Cancer Survivorship Needs Assessment. The unmet needs of cancer survivors in Ireland: A scoping review 2019. Cork: National Cancer Registry Ireland; 2019. Disponible https://www.ncri.ie/publications/researchreports/unmet-needs-cancer-survivors-irelandscoping-review-2019

12. Canadian partnership against cancer. Living with Cancer. A Report on the Patient experience. Toronto: Canadian Partnership Against Cancer Corporation; 2018. Disponible en:

https://s22457.pcdn.co/wp-

content/uploads/2019/01/Living-with-cancerrepo

t-patient-experience-EN.pdf

13. Institute of Medicine (IOM). From Cancer Patient to Cancer Survivor: Lost in Transition. Washington, DC: The National Academies Press; 2005.

14. Sociedad Española de Oncología Médica (SEOM). Manifiesto SEOM Sobre La Atención a Los Largos Supervivientes de Cáncer, http://www.seom.org/en/publicaciones/publica ciones-

seom/largossupervivientes/\%0D103911-

manifiesto-seom-sobre-la-atencion-a-loslargossupervivientes-\%0Dde-cancer; 2015[consultada 15/09/2020].

15. Sociedad Española de Oncología Médica (SEOM). Plan Integral de Atención a Los Largos Supervivientes de Cáncer, https://seom.org/seomcms/images/stories/rec ursos/PLAN_INTEGRAL_LARGO_SUPERVIVIENT E.pdf; 2013 [consultada $15 / 09 / 2020$ ].

16. Nekhlyudov L, O'malley DM, Hudson S V. Integrating primary care providers in the care of cancer survivors: gaps in evidence and future opportunities. Lancet Oncol 2017;18(1):e30e38. doi:10.1016/S1470-2045(16)30570-8

17. Garcia-Vivar C, Elizondo N, Ambrosio L. Primary Care Nursing Is Essential to Fully Implement Survivorship Care Plans for Longterm Cancer Survivors and Their Families. Cancer Nurs 2019;42(3):177-8. doi:10.1097/NCC.0000000000000704

18. Departamento de Salud de Navarra. Plan de Salud de Navarra 2014-2020. Estrategia de prevención y atención al cáncer. https://www-navarra--

es.insuit.net/home_es/Temas/Portal+de+la+S alud/Ciudadania/Nuevo+Modelo+asistencial/PI an+Salud+Navarra/Plan+de+Salud+de+Navar $\mathrm{ra}+2014-$

$2020+$ Profesionales/Estrategias+y+Programas /Prevencion+y+Atencion+al+Cancer/ [consultada 15/09/2020].

19. Departamento de Salud del Gobierno Vasco. Plan Oncológico de Euskadi 2018-2023, 
https://www.euskadi.eus/contenidos/informaci on/plan_oncologico_2018_2023/es_def/adjunt os/plan-oncologico-euskadi-2018-2023.pdf;

2018 [consultada 15/09/2020].

20. Conselleria de Sanitat. Generalitat Valenciana. Estrategia contra el cáncer de la Comunitat Valenciana 2019-2022, https://socvalped.com/wpcontent/uploads/2019/06/EstrategiaCancer201 90121.pdf ; 2019 [consultada 15/09/2020].

21. Generalitat de Catalunya. Pla contra el càncer a Ctalunya 2015-2020, http://hdl.handle.net/11351/1685 [consultado 20/10/2020]

22. Sacyl. Junta de Castilla y León. Estrategia Regional de Atención al Paciente Oncológico en Castilla y León ONCYL, https://www.saludcastillayleon.es/institucion/e s/atencion-paciente-oncologico; 2019[consultada 15/09/2020].

23. Hewitt M, Greenfield S, Stovall E. From Cancer Patient to Cancer Survivor: Lost in Transition. Washington DC: The National Academies Press; 2006.

24. Rowland JH, Kent EE, Forsythe LP, et al. Cancer survivorship research in Europe and the United States: Where have we been, where are we going, and what can we learn from each other? Cancer 2013;119:2094-2108. doi: $10.1002 /$ cncr. 28060

25. Joo JY, Liu MF. Effectiveness of Nurse-Led Case Management in Cancer Care: Systematic Review. Clin Nurs Res. 2019 Nov;28(8):968991. doi: $10.1177 / 1054773818773285$. Epub 2018 May 4. PMID: 29726271

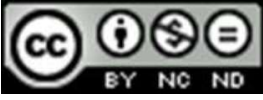

